\title{
GCU
}

Glasgow Caledonian

University

University for the Common Good

\section{Investigating effects of emoji on neutral narrative text: evidence from eye movements and perceived emotional valence}

Robus, Christopher M.; Hand, Christopher J.; Filik, Ruth; Pitchford, Melanie

Published in:

Computers in Human Behavior

DOI:

10.1016/j.chb.2020.106361

Publication date:

2020

Document Version

Author accepted manuscript

Link to publication in ResearchOnline

Citation for published version (Harvard):

Robus, CM, Hand, CJ, Filik, R \& Pitchford, M 2020, 'Investigating effects of emoji on neutral narrative text: evidence from eye movements and perceived emotional valence', Computers in Human Behavior, vol. 109, 106361. https://doi.org/10.1016/j.chb.2020.106361

\section{General rights}

Copyright and moral rights for the publications made accessible in the public portal are retained by the authors and/or other copyright owners and it is a condition of accessing publications that users recognise and abide by the legal requirements associated with these rights.

Take down policy

If you believe that this document breaches copyright please view our takedown policy at https://edshare.gcu.ac.uk/id/eprint/5179 for details

of how to contact us. 
Investigating effects of emoji on neutral narrative text:

Evidence from eye movements and perceived emotional valence

Christopher M. Robus ${ }^{1 *}$, Christopher J. Hand ${ }^{2}$, Ruth Filik $^{3} \&$ Melanie Pitchford ${ }^{1}$

${ }^{1}$ School of Psychology, University of Bedfordshire, Luton, United Kingdom

${ }^{2}$ Department of Psychology, Glasgow Caledonian University, Glasgow, United Kingdom

${ }^{3}$ School of Psychology, The University of Nottingham, Nottingham, United Kingdom

\section{*Corresponding Author}

Christopher Robus

School of Psychology

University of Bedfordshire

University Square, Luton, LU1 3JU

Email: christopher.robus@study.beds.ac.uk

Telephone: +44 (0)1582400400 
RUNNING HEAD: EMOJI EFFECTS ON READING AND SENTENCE RATING

1

2 Digital images of faces such as emoji in virtual communication have become increasingly popular, but current research findings are inconsistent regarding their emotional effects on

4 perceptions of text. Similarly, emoji effects on reading behaviours are largely unknown and 5 require further examination. The present study $(N=41)$ investigated how the position and 6 emotional valence of emoji in neutral narrative sentences influenced eye movements during

7 reading and perceptions of sentence valence. Participants read neutral narrative sentences containing smiling or frowning emoji in sentence-initial or sentence-final positions and rated

the perceived emotional valence of the sentence. Results from linear mixed-effects models demonstrated significantly longer fixations on sentence-final emoji and longer sentence reading times when emoji were in sentence-final positions. These findings are comparable to sentence 'wrap-up' effects witnessed in the processing of lexical units during sentence reading, providing new evidence towards the way readers integrate emoji into contextual processing. However, no impact of emoji valence or position on first-pass target word processing or sentence-valence ratings were found. This would refute previous suggestions that digital faces influence text valence, raising questions about reader preference for emoji or sentence sentiment, the influence of sentence formatting, and delivery/display mechanism on these effects.

Keywords: emoji; reading; eye movements; valence; emotion

\section{Funding sources}

This research did not receive any specific grant from funding agencies in the public, commercial, or not-for-profit sectors. 
EMOJI EFFECTS ON READING AND SENTENCE RATING

\section{Background}

Increased access to the online world through mobile technology has allowed people to communicate with others almost instantly. As a result, online communicative behaviours have evolved to adapt to restrictions within these applications and written communication itself. Different forms of textism serve a function in virtual communication; for example, the use of text-speak shortcuts were originally designed to limit character usage during text messaging (Kemp, 2010). However, one notable addition is the introduction of digital facial representations, such as the emoticon (textual sequences designed to look like faces from a specific angle, e.g., :-), :-D, :-o ; Filik et al., 2016; Kaye, Wall, \& Malone, 2016) and its successor the emoji (image-based Unicode symbols, e.g., 2 , 目; Kaye, Malone, \& Wall, 2017). Originally introduced as a way of displaying basic emotions in digital messages, their uses are now more commonly likened to non-verbal cues and gestures within face-to-face interactions (Gawne \& McCulloch, 2019; Lo, 2008).

\subsection{Digital facial representations and text-processing}

The investigation of digital faces and their effects on the emotional perceptions of textual messages is a well-researched area. One of the earliest examples of psychological research investigating the influence of digital faces on text are the experiments of Walther and D'Addario (2001). In their paradigm, they asked participants to rate a series of artificially created emotive emails which contained either positive (e.g., smiling) or negative (e.g., frowning) emoticons. They found that perceptions of text valence were not influenced by the emoticons, except in cases when negative emoticons were placed within a negative email.

This indicated that the emotional influence of emoticons on a message can be overshadowed by the sentiment of the text in some cases, whereas negative emoticons can reinforce the sentiment of negative text. 


\section{EMOJI EFFECTS ON READING AND SENTENCE RATING}

However, replications of this effect have been far from consistent. For example, Derks, Bos, and Von Grumbkow (2008) replicated the paradigm of Walther and D'Addario, and found that emoticons strengthened both positive and neutral messages towards their respective valence, but did not detect the negativity effect found in Walther and D'Addario's original study. On the other hand, Lo (2008) presented emotional textual conversations to a sample of instant message service users; these were presented either as pure text or with one of a number of positive and negative emoticons after the sentence. These conversations were then rated by receivers for perceived emotion, attitude and attention. They found that the inclusion of emoticons influenced perceptions and strengthened the sentimental intent of all messages, biasing them towards the valence of the emoticon. Ultimately, the lack of cohesion in these findings and their notable inconsistencies warrant further investigation to understand the nature of these effects. As for emoji, which share the same underlying concept but are graphically different from emoticons, present literature examining their sentimental effects is limited.

$$
\text { Many studies have focused on conversational formats of language that involve }
$$
dialogues with responses, as these are often the most typical examples of written text that include facial representations (Riordan \& Kreuz, 2010; Rodrigues, Lopes, Prada, Thompson, \& Garrido, 2017; Skovholt, Grønning, \& Kankaanranta, 2014). However, this raises questions about whether emoji can have an effect on other forms of statement, such as sentences outlining a narrative of an event from an external perspective (i.e. third-person narration). Willoughby and Liu (2018) conducted a factorial experiment to assess the impact of emoji use and narrative versus non-narrative conversational formats on the processing of health text message interventions. A sample of college students viewed screenshots of iMessage conversations containing either narrative or non-narrative sentences, which included either no emoji, a low frequency (one emoji) or a high frequency of emoji (three 


\section{EMOJI EFFECTS ON READING AND SENTENCE RATING}

emoji) with the messages. Their findings showed mixed results on measures of message elaboration, credibility, attention and personalisation, with non-narrative messages without emoji eliciting higher levels of message credibility and elaboration, and messages with higher quantities of emoji drawing greater attention, regardless of narrative format. On a societal level, emoji are being used in a much wider context than in digital communication, in some cases to the extent of being used as a form of delivering a narrative story in themselves. For example, in 2009, the literary classic 'Moby Dick' was famously translated into emoji, entitled ‘Emoji Dick' by Fred Benenson. However, while emoji may influence perceptions of communicative text, little is known about how they could impact other domains where they are currently being used, such as narrative sentences. The Social Information Processing theory (Walther, 1992) suggests that the use of cues, which digital faces would be categorised as, in computer-mediated communication is motivated by a desire to form and maintain relationships with another user (Rodríguez-Hidalgo, Tan, \& Verlegh, 2017). In consideration of this point, it is plausible that the function of digital faces and their subsequent effects on a receiver are context-dependent, with a reduced impact outside of the realm of online interactive communication. Such findings would have an impact on the use of emoji in a wider context outside of online communication, such as business advertisements, political campaigns and education.

\subsection{Emoji and lexical-semantic processing}

By their nature, digital emblems that represent emotionally expressive faces retain their own semantic properties, including the emotion that they portray. They are often compared to the role of facial expressions and other non-verbal emotional cues in offline communication. As such, the way that they interact with accompanying text and influence reading has theoretical ramifications for the field of psycholinguistics, indicating how readers 


\section{EMOJI EFFECTS ON READING AND SENTENCE RATING}

begin to decode emoji and integrate them into the context of the sentence. However, the lexical-semantic effects of emoji are remarkably underexplored in the literature. If emoji function in written discourse in similar ways to non-verbal cues in offline face-to-face communication, and contain their own linguistic and semantic properties that enable readers to integrate them into the context of the accompanying message, then it should be expected that they will influence the way a reader processes text. Evidence from event-related potential research has demonstrated that neural responses to sentences with congruent, incongruent and ironic emoji elicit strong responses which parallel the processing of irony and lexical predictability (Weissman \& Tanner, 2018), as well as possible priming effects of emoji on text (Comesaña et al., 2013). Similarly, research using on-line emotional measures (e.g. electrodermal activity, facial electromyography) to assess physiological responses to assess ironic language and emoticons has provided insights into how readers respond to sentences with digital faces (Thompson, Mackenzie, Leuthold, \& Filik, 2016). However, to our knowledge, no research at this point has utilised measures of on-line eye movements (see Rayner, 2009 for a review) during the reading of sentences containing emoji, which can provide highly valuable and naturalistic time-based response data (Sereno \& Rayner, 2003). The processing of sentences is contingent on a number of factors, predominantly the grammar and syntactic structures of the respective language. The order of words in a sentence can heavily impact how the sentence is perceived and parsed, with deviations or ambiguity resulting in increased difficulty (Rayner, Carlson, \& Frazier, 1983). However, as a relatively new construct, emoji do not necessarily have set grammatical or syntactic rules that are regularly followed. As such, the impact of the spatial position of digital faces in sentences on perceptual and attentional behaviours is largely unknown. Predominantly, research focus in this area has been placed on establishing where users choose to place their faces in a sentence. According to evidence provided from big data analyses, in approximately half of 


\section{EMOJI EFFECTS ON READING AND SENTENCE RATING}

cases users choose to place their emoji or emoticons at the end of a sentence, although this may be context-dependent on the function of the responding message (Garrison, Remley, Thomas, \& Wierszewski, 2011; Tauch \& Kanjo, 2016). Amaghlobeli (2012) and Spina (2018) suggest that this sentence-final placement is deliberate rather than random and demonstrates the function of digital faces as structural markers in linguistic processing (e.g. indicating boundaries in clauses and sentences) in a comparable way to punctuation marks. However, these findings do not address what effect this positioning has on a receiver of a message, nor whether this placement has a beneficial or detrimental effect on cognitive processing. As such, the impact of emoji position on the reading of: words within a sentence, the whole of the sentence, and the emoji itself, remains unknown. As a widely used entity across cultures, this predominant placement of emoji at the end of the sentence must arguably serve a function in lexical processing or else it would not occur so commonly.

Word-position effects have been previously documented in psycholinguistic research, demonstrating differences in processing of words at the beginning and end of a sentence compared to those in the centre. Furthermore, evidence suggests that readers spend longer fixating on sentence-final information than sentence-initial or words in the middle of the sentence (e.g., Kuperman, Dambacher, Nuthmann, \& Kliegl, 2010; Warren, White, \& Reichle, 2009). One explanation for this processing cost of sentence-final information comes in the form of 'wrap-up' effects in sentence processing, which concern higher-order processes of comprehension and semantic integration of accumulated information from preceding words. Conversely, the reading of sentence-initial information encompasses 'startup' effects that are vital for oculomotor planning of saccadic eye movements across the remainder of the sentence. In accordance with serial theories of ocular control during reading, such as the E-Z Reader model (see Reichle \& Sheridan, 2015), lexical units within the boundaries of the fovea are initially processed in isolation from the surrounding words, while 


\section{EMOJI EFFECTS ON READING AND SENTENCE RATING}

the process of making a forward or regressive saccade can be influenced by a number of other factors (e.g., word frequency, contextual predictability; Hand, Miellet, O’Donnell, \& Sereno, 2010; Rayner, 1998). However, wider global sentence comprehension requires more complex integrations of accumulated semantic information once reading is complete, which incurs a cost in processing speed (Balogh, Zurif, Prather, Swinney, \& Finkel, 1998; Hirotani, Frazier, \& Rayner, 2006; Kuperman et al., 2010; Payne \& Stine-Morrow, 2012; Warren et al., 2009). The theoretical concept of sentence wrap-up has only been attributed to the processing of clause- and sentence-final words and in some cases the inclusion of punctuation. However, it has never been attributed to wider linguistic entities, such as emblems representing digital faces. Although emoji may not have the same linguistic structures as words, they do retain their own semantic value. It is possible that readers apply higher-order processing on emoji at the end of in a sentence, when compared to sentence-initial positioning, to assist in the semantic integration of the emblem and the sentence. This would have interesting theoretical implications for current psycholinguistic understanding of sentence processing, demonstrating that higher-order semantic processing during reading can extend to units that are not natural words.

\subsection{The current study}

On the basis of the previously discussed literature and with the gaps in theoretical knowledge established, the current study investigated whether the spatial position and expressive valence of emoji in a sentence affect the reading and perceptions of accompanying neutral narrative English sentences. To assess this, eye movements were recorded during the reading of sentences containing emoji at the beginning or end of a sentence, with readers subsequently rating how emotionally valent they perceived the sentences to be. To evaluate whether emoji valence and position influenced the reading of individual words in the centre 


\section{EMOJI EFFECTS ON READING AND SENTENCE RATING}

of the sentence, fixations on controlled centre-positioned target words were recorded.

Furthermore, to investigate these effects on wider sentence-level reading, total sentence reading durations were also measured.

As demonstrated, previous research designs examining the effects of digital faces on emotionally neutral text have included them as a level of an experimental manipulation, focusing heavily on the impact on emotional sentences. However, one could argue that these designs confound the potential effect, as readers are primed to decode the neutral sentence trials in an explicitly emotional way as a result of the other positive- and negative-sentence trials. The focus of the current study is to control for this by only using neutral sentences, and attempt to address the true nature of the effect of digital faces on emotionally neutral text. Similarly, given the current study's predominantly exploratory nature in terms of emoji position and narrative statements, it is hoped that this study will act as a knowledge-base for future research to develop upon.

If digital faces do have a similar function to non-verbal cues and have their own pseudo-linguistic properties (Lo, 2008), then their position could impact the processing of the sentence, words in the centre of the sentence, and the emoji itself. More specifically, if higher-order processes involving the semantic integration of emoji and sentences incur a 'wrap-up' cost during reading, then this will be reflected in the eye movement data. As such, it is hypothesised that 'fixations on emoji will be significantly longer when emoji are in a sentence-final position'. On the other hand, although the reading of sentence-initial and sentence-final words can be differentiated from centre-position words (Kuperman et al., 2010), concrete predictions of differences as a result of emoji positions and valence effects are more problematic due to a lack of prior evidence. It is possible that readers may fixate for longer on centre-position words or make more visual regressions dependent on the positioning of the emoji, but the nature and direction of this cannot be ascertained without 


\section{EMOJI EFFECTS ON READING AND SENTENCE RATING}

existing baseline data. As a result, the research question 'what are the effects of emoji valence and position on centre-position words and total sentence reading times?' was created and assessed through quantitative analyses on word-level and sentence-level fixation measures. In addition, given the inconsistent findings regarding perceived emotional valence effects and potential questions about the appropriateness of non-communicative use of emoji, the broader research question 'what are the effects of emoji position and valence on perceptions of emotional valence?' was generated and assessed via analyses on the ratings of sentence emotional valence during the experiment.

\section{$\underline{\text { 2. Method }}$}

2.1 Design

To control for potential individual differences in reading speeds and capabilities, a fully factorial $2 \times 2$ within-subjects design was applied. The valence (smiling, frowning) and position (sentence-initial, sentence-final) of the emoji in the sentence were manipulated. Standard measures of eye movement behaviour (Rayner, 1998, 2009) were calculated from areas of interest (AOIs) on the region containing the emoji and a target word region (which consisted of a five-letter target word and the space immediately preceding the target word). These included: first fixation durations (the duration of the first fixation in an AOI during first-pass reading); single fixation durations (the duration when only one fixation is made in an AOI); gaze durations (the sum of fixation durations in an AOI during first-pass reading); total fixation durations (the sum of all fixation durations in an AOI) and fixation counts (number of fixations in an AOI). Similarly, total sentence reading duration (sum of all fixation durations within the sentence) was measured, both including and excluding fixations in the emoji region. For an example of the AOI analysis regions, please see Figure 1. The 
EMOJI EFFECTS ON READING AND SENTENCE RATING

224

225

226

227

228

229

230

231

232

233

234

235

236

237

238

240

241

242

perceived emotional valence of target sentences was also measured on a 1-to-9 rating scale (1=highly negative; $9=$ highly positive).



Figure 1. Example of target word, emoji and sentence for a positive sentence-final emoji stimulus.

\subsection{Participants}

Participants ( $N=44 ; 34$ females) were native English readers aged between 18 and 59 years old $\left(M_{\mathrm{age}}=28.85\right.$ years, $\left.S D_{\text {age }}=12.97\right)$ with normal or corrected-to-normal vision and no diagnoses of reading disorders. Recruitment employed advertisement and convenience sampling methods. All participants were entered in a prize draw for a $£ 25$ Amazon UK voucher, and undergraduate students $(n=25)$ were offered course credit for participation. Prior to completing the experiment, participants were asked to self-report their general emoji use and exposure. Four participants reported always using emoji in the messages they composed and 21 reported using emoji 'most of the time', with the remainder using emoji 'half of the time' or 'sometimes'. On average, participants estimated using emoji in $60 \%$ of their messages $(S D=28.3 \%)$. In terms of receiving messages with emoji, 20 reported receiving them 'most of the time', 17 reporting 'about half of the time', and the remainder reporting 'sometimes', with an average of $61 \%$ of received messages reported as 


\section{EMOJI EFFECTS ON READING AND SENTENCE RATING}

containing an emoji $(S D=20.1 \%)$. All participants reported using and receiving more positively-valent emoji than negative.

\subsection{Materials and Apparatus}

Thirty-six single line narrative sentences were created as stimuli. Sentences were composed in third-person perspective and were neutral in emotional valence. Stimulus neutrality was assessed by an independent group of anonymous online participants $(N=62)$, who were presented the experimental stimuli (without any emoji characters) and filler material in a random order. They were then asked to rate them on a scale of one to nine for how emotionally valent they thought they were. The mean sentence valence ratings were considered within the appropriate parameters of neutrality $\left(M_{\text {valence }}=5.13, S D_{\text {valence }}=0.54\right.$, $\min =4.14, \max =5.94)$. Sentence length ranged from 66 to 75 characters $\left(M_{\text {length }}=69.89\right.$ characters, $S D_{\text {length }}=2.03$ ). For the full list of the sentences and individual rating scores, please refer to Appendix A.

Regarding the emoji used in the study, previous research has suggested that demographic factors, such as cultural background, gender and age, can influence how digital faces are applied and interpreted (Fullwood, Orchard, \& Floyd, 2013; Oleszkiewicz et al., 2017; Wolf, 2000). To control for possible bias as a result of these factors, two emoji were selected for the study that were comparable in colour and formatting but could be differentiated by their expressions. As such, the 'slightly smiling face' ( $\because$; U+1F642) was chosen for positive valence conditions, and the 'slightly frowning face' ( $\because$; U+1F642) for negative valence. Both were presented in Twitter's open source 'Twemoji' format, in full colour and in a comparable size to the text.

Additionally, each sentence contained a five-letter target word positioned towards the centre of the sentence, and an emoji was placed either before or after the sentence. These 


\section{EMOJI EFFECTS ON READING AND SENTENCE RATING}

271

272

273

274

277

278

target words were nouns carefully chosen to avoid having explicit (e.g. name of an emotion) or implicit emotional context (e.g. names of colours). Target words were assessed for frequency of occurrence ${ }^{1}\left(M_{\text {freq }}=24322.11, S D_{\text {freq }}=30840.63\right.$, min $=3314$, max $\left.=183044\right)$, arousal $\left(M_{\text {arousal }}=4.86, S D_{\text {arousal }}=1.19, \min =3.17, \max =7.77\right)$ and valence $\left(M_{\text {valence }}=5.89\right.$, $\left.S D_{\text {valence }}=0.95, \min =4.09, \max =8.15\right)^{2}$. Frequency of occurrence was taken from the SUBTLEX-UK database of British-based television subtitles (van Heuven, Mandera, Keuleers, \& Brysbaert, 2014), whereas target arousal and valence ratings were taken from The Glasgow Norms database (Scott, Keitel, Becirspahic, Yao, \& Sereno, 2019). For example target stimuli, please refer to Table 1 (a full list of stimuli and target specifications are presented in Appendix A).

Table 1: Examples of experimental materials

\begin{tabular}{|c|c|c|}
\hline Valence & Position & Example \\
\hline \multirow[t]{2}{*}{ Positive } & Initial & $\because$ When the guest returned to the hotel later there was nobody to be seen \\
\hline & Final & Steven waited at home for his order to be delivered in the morning $\because$ \\
\hline \multirow[t]{2}{*}{ Negative } & Initial & $\because$ Jenny started dressing for the party when there was a knock on the door \\
\hline & Final & Charlotte returned to her private study and started working on her essay $\because$ \\
\hline
\end{tabular}

Note. Target words presented in italics above, but were non-italicised during experimental sessions.

\footnotetext{
${ }^{1}$ Frequency of occurrence is how often a specific word appears in its respective language based on a collected corpus of data. For example, the SUBTLEX-UK frequency scores outline the number of times a specific word appears in a corpus of 200 million words taken from British subtitles.

${ }^{2}$ The Glasgow Norms database measured both arousal and valence on a 9-point scale with a centre score representing moderate arousal or emotional neutrality respectively.
} 


\section{EMOJI EFFECTS ON READING AND SENTENCE RATING}

A Latin-square design was used to counter-balance sentences and the conditions they represented. This involved a rotation of the emoji valence-position combinations for each stimulus, with participants being randomly allocated to different sets. All 36 sentences were presented to participants, but participants saw each sentence in only one of the possible manipulation combinations. Thirty-six additional neutral, third-person narrative sentences containing no emoji were added as filler material to deter demand characteristics but were removed ahead of statistical analysis. With the addition of three practice trials, this totalled 75 trials.

A desktop-mounted SR Research EyeLink 1000 eye tracker was used to record eye movements with a spatial resolution of $0.01^{\circ}$ at a sampling rate of $1000 \mathrm{~Hz}$. Eye movements were recorded using pupil tracking and corneal reflections. Although viewing was binocular, recordings were taken from the right eye. Head movements were minimised by using a chin and forehead rest. Instructions and stimuli were presented on an Iiyama ProLite B1906S monitor (1280 x 1024, $60 \mathrm{~Hz})$ through SR Research Experiment Builder software (v 2.2.1). At a viewing distance of $104 \mathrm{~cm}, 1^{\circ}$ of visual angle equated to 3.1 characters of text. Screen brightness was adjusted for the comfort of each participant and thereafter held constant. A desktop keyboard was used to enter emotional valence rating scores and progress through the experiment. Stimuli were presented in black, 16-point non-proportional Courier New font on a white background.

\subsection{Procedure}

Approval for the study was granted by the [HOST INSTITUTION] School of Psychology ethics committee. Participants were given an information sheet prior to participation, provided informed consent and received a full debrief following the experiment. The eye tracker was calibrated using a 7-point procedure, followed by a 9-point 


\section{EMOJI EFFECTS ON READING AND SENTENCE RATING}

validation check for tracking error. Each calibration presented fixation points serially along the horizontal and vertical edges of the screen. In the validation checks, average error was below $0.30^{\circ}$ and fixation point maximal error was below $0.60^{\circ}$. Instructions for calibration and trial procedures were provided at two points (before and after practice trials) prior to the experimental trials.

Participants completed a three-trial practice set, followed by the experimental and filler trials. The practice trials included one example of a sentence-initial, sentence-final and filler sentence to familiarise the reader with the format of the stimuli. In each trial, a driftcorrection point was used first to assess calibration quality before each trial. When calibration discrepancies were observed, the tracker was recalibrated. A fixation point $(+)$ marking the position of either the first character in the sentence (sentence-final conditions) or a sentenceinitial emoji would then be presented in the top-left corner of the monitor. Fixating on this point would present the sentence to the participants. Participants were instructed to read the sentences carefully, silently, and at their own pace. The presentation order of sentences was randomised for each participant. When finished, they looked in the bottom-right corner of the monitor to indicate the end of reading. Participants were then presented with a screen asking them to rate the emotional valence of the sentence they had just read on a scale of 1 (highly negative) to 9 (highly positive) with 5 being neutral. Participants were instructed to enter their response through the computer keyboard in front of them.

\footnotetext{
$\underline{\text { 3. Results }}$

The chosen AOIs were the region the emoji was positioned - either at the beginning (sentence-initial) or end (sentence-final) of the sentence - and the region containing the midsentence target word. Data from three participants was removed during preliminary data assessment due to excessive tracking loss, resulting in a final sample of 41 participants.
} 


\section{EMOJI EFFECTS ON READING AND SENTENCE RATING}

340 Fixations shorter than $100 \mathrm{~ms}$ were merged if within 1.5 characters of another fixation or were otherwise removed. The upper cut-off parameter for individual fixation durations was $800 \mathrm{~ms}$.

342 Blinks in the critical AOIs (i.e., target word and emoji) were also removed from analysis.

343 Overall, this accounted for an exclusion of $4.9 \%$ of the data. AOIs containing no fixations 344 were treated as empty cells in the analyses.

Fixation durations were analysed using linear and generalised linear mixed effects modelling via the lme4 package (Bates, Mächler, Bolker, \& Walker, 2015) and ordinal linear mixed models through the ordinal package (Christensen, 2019) within the R statistical environment (3.6.1) using the R Studio Desktop software extension (1.2.1335). Mixed-model analyses have become a preferred method of analysis for within-subjects data, as they are robust to violations in assumptions such as the independence of observations assumption. The full linear mixed-effects models contained the two independent within-subject variables (valence, position) as fixed effects, the interaction term, and the random-effects structure. A data-driven approach was taken to estimate the appropriate random-effects structure for each model (for a summary of the random-effects structures, please see Appendix B). Significance values for fixed effects were generated using likelihood-ratio tests comparing the full statistical model against reduced models that remove either the main effects of emoji valence, position or the interaction effect. The random-effects structures were maintained across the likelihood-ratio tests.

\subsection{Emoji Region}

The residual plots for the fixation measures within the emoji region indicated breaches in residual normality; therefore, logarithm transformations were applied at base 10 to the breached outcome variables. For a summary of descriptive statistics, please refer to Table 2. 
EMOJI EFFECTS ON READING AND SENTENCE RATING

365

366

367 368

Table 2: Means, standard deviations and confidence intervals of measures by conditions

\begin{tabular}{cccccc}
\hline AOI & \multicolumn{2}{c}{ Sentence-initial } & \multicolumn{2}{c}{ Sentence-final } \\
Emoji & Obs & Negative & Positive & Negative & Positive \\
\hline FFD & 688 & $216(47)$ & $217(56)$ & $296(157)$ & $304(139)$ \\
SFD & 606 & $215(39)$ & $217(56)$ & $300(160)$ & $309(143)$ \\
GD & 688 & $222(79)$ & $217(56)$ & $319(193)$ & $319(156)$ \\
TD & 801 & $315(218)$ & $313(166)$ & $407(290)$ & $382(211)$ \\
FC & 1476 & $0.80(.80)$ & $0.76(.72)$ & $0.62(.78)$ & $0.61(.77)$ \\
\hline Target & Obs & & & & \\
FFD & 889 & $232(77)$ & $247(90)$ & $239(87)$ & $241(78)$ \\
SFD & 786 & $238(78)$ & $248(93)$ & $242(89)$ & $242(77)$ \\
GD & 889 & $253(97)$ & $258(111)$ & $261(126)$ & $256(99)$ \\
TD & 1042 & $346(233)$ & $351(265)$ & $366(277)$ & $368(238)$ \\
FC & 1476 & $0.89(1.05)$ & $0.83(.99)$ & $1.29(1.13)$ & $1.31(.98)$
\end{tabular}

\begin{tabular}{|c|c|c|c|c|c|}
\hline Sentence & & & & & \\
\hline $\begin{array}{l}\text { TSRD (inc. } \\
\text { emoji) }\end{array}$ & 1475 & $3856(1801)$ & 3766 (1889) & $4053(2061)$ & $3930(1930)$ \\
\hline $\begin{array}{l}\text { TSRD (exc. } \\
\text { emoji) }\end{array}$ & 1475 & $3662(1750)$ & $3574(1822)$ & $3862(2027)$ & 3748 (1780) \\
\hline Valence Score & 1476 & $5.35(1.63)$ & $5.20(1.49)$ & $5.18(1.60)$ & $5.27(1.57)$ \\
\hline \multicolumn{6}{|l|}{$95 \% \mathrm{CI}$} \\
\hline \multicolumn{6}{|l|}{ Emoji } \\
\hline FFD & & $(212,220)$ & $(213,221)$ & $(284,308)$ & $(294,314)$ \\
\hline SFD & & $(212,218)$ & $(213,221)$ & $(287,313)$ & $(298,320)$ \\
\hline GD & & $(216,228)$ & $(213,221)$ & $(305,333)$ & $(307,331)$ \\
\hline $\mathrm{TD}$ & & $(300,330)$ & $(302,325)$ & $(387,427)$ & $(367,397)$ \\
\hline $\mathrm{FC}$ & & $(0.76,0.84)$ & $(0.72,0.80)$ & $(0.58,0.66)$ & $(0.57,0.65)$ \\
\hline \multicolumn{6}{|l|}{ Target } \\
\hline FFD & & $(227,237)$ & $(241,253)$ & $(233,245)$ & $(236,246)$ \\
\hline SFD & & $(233,243)$ & $(242,255)$ & $(236,248)$ & $(237,247)$ \\
\hline GD & & $(247,259)$ & $(251,265)$ & $(253,269)$ & $(249,263)$ \\
\hline $\mathrm{TD}$ & & $(332,360)$ & $(335,367)$ & $(349,383)$ & $(354,383)$ \\
\hline $\mathrm{FC}$ & & $(0.84,0.94)$ & $(0.78,0.88)$ & $(1.23,1.35)$ & $(1.26,1.36)$ \\
\hline \multicolumn{6}{|l|}{ Sentence } \\
\hline TSRD (no emoji) & & $(3760,3950)$ & $(3760,3860)$ & $(3950,4160)$ & $(3830,4030)$ \\
\hline $\begin{array}{l}\text { TSRD (with } \\
\text { emoji) }\end{array}$ & & $(3570,3750)$ & $(3480,3670)$ & $(3760,3970)$ & $(3660,3840)$ \\
\hline
\end{tabular}


EMOJI EFFECTS ON READING AND SENTENCE RATING

\begin{tabular}{lllll} 
Valence Score & $(5.27,5.43)$ & $(5.12,5.28)$ & $(5.10,5.26)$ & $(5.19,5.35)$ \\
\hline
\end{tabular}

Note. $\mathrm{AOI}=$ area of interest $; \mathrm{Obs}=$ number of observations; FFD = first fixation duration, $\mathrm{SFD}=$ single fixation duration, $\mathrm{GD}=$ gaze duration, $\mathrm{TD}=$ total fixation duration, $\mathrm{FC}$ = fixation count, TSRD $=$ total sentence reading duration (including or excluding emoji AOI fixations included). Fixation duration measures rounded to nearest whole number; Valence Scores rounded to 2DP. Standard deviations of means are presented in parentheses.

The results (see Table 3) indicated that emoji positioned at the end of the sentence (sentence-final) had longer first-pass and late stage fixations than those positioned at the beginning of the sentence (sentence-initial). Specifically, significant main effects of emoji position were detected on first fixation durations, single fixation durations, gaze durations and total fixation durations. For a visual representation of these findings, please see Figure 1. Similarly, generalised mixed models showed a significant main effect of emoji position on fixation counts in the emoji region, with sentence-initial emoji drawing more fixations than sentence-final (see Tables 2 and 3). However, there were no significant main effects of emoji valence on fixations in the emoji region, indicating that whether the emoji exhibited an emotionally positive or negative expression did not result in differing fixation durations.

Similarly, the emoji position $\times$ emoji valence interaction effects were non-significant for all fixation duration measures in the emoji interest region. 

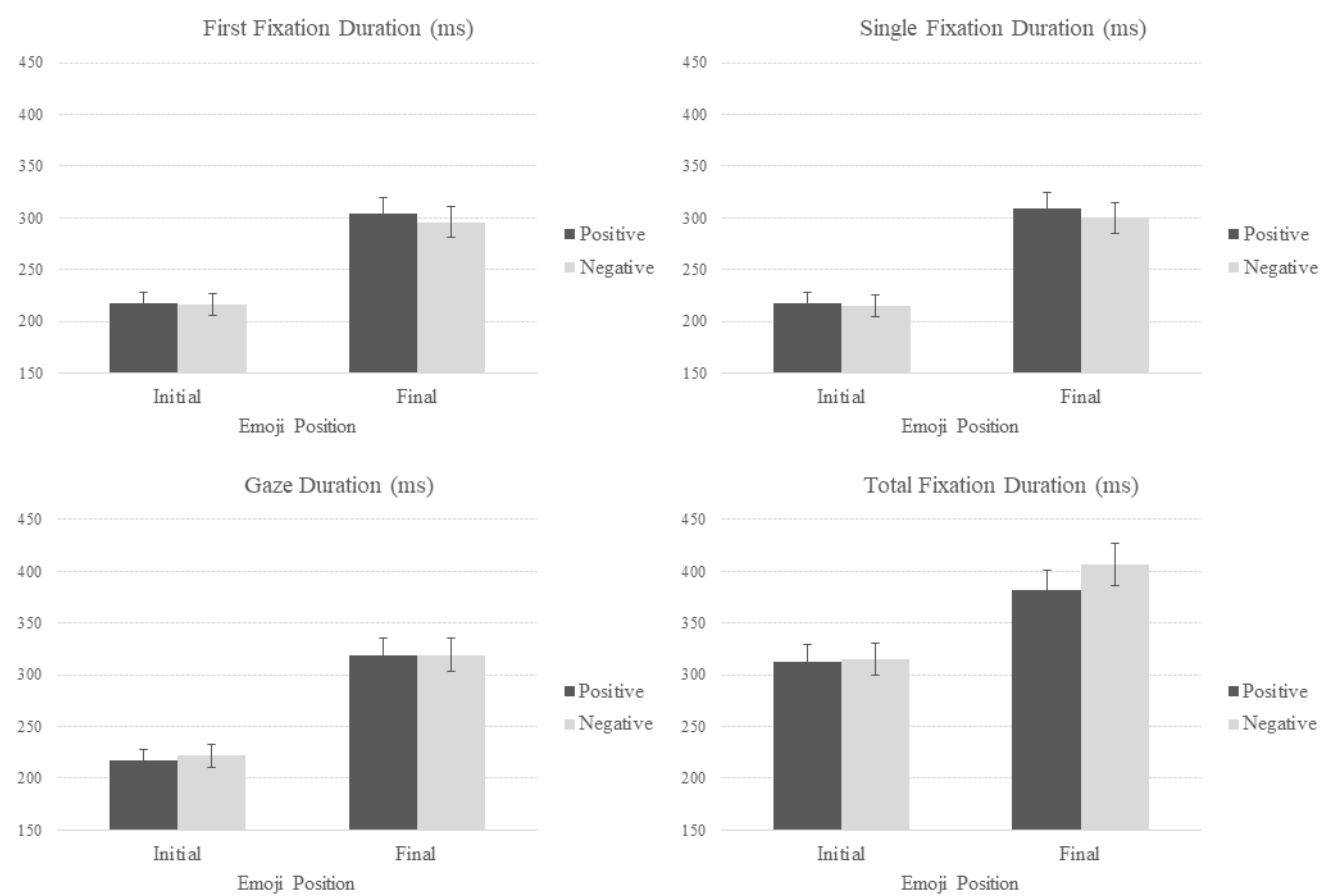

393

394

395

396

397

398

399

Figure 2. Mean first fixation, single fixation, gaze and total fixation durations in emoji region in positive and negative sentence-initial and sentence-final position conditions.

Table 3: Results of linear mixed effects models and likelihood-ratio tests

\begin{tabular}{|c|c|c|c|c|c|c|c|}
\hline \multirow[b]{2}{*}{ AOI } & \multirow[b]{2}{*}{ Measure } & \multirow[b]{2}{*}{ Effect } & \multicolumn{3}{|c|}{ LMEs } & \multicolumn{2}{|c|}{ Likelihood-Ratio } \\
\hline & & & $b$ & $S E$ & $t$ & $\chi^{2}$ & $p$ \\
\hline \multirow[t]{13}{*}{ Emoji } & FFD & Valence & .002 & .016 & 0.10 & 1.12 & .29 \\
\hline & & Position & .094 & .016 & 5.90 & 75.52 & $<.001$ \\
\hline & & Val $\times$ Pos & .020 & .023 & 0.90 & 0.80 & .37 \\
\hline & SFD & Valence & .001 & .016 & 0.03 & 0.67 & .41 \\
\hline & & Position & .101 & .017 & 6.08 & 80.13 & $<.001$ \\
\hline & & Val $\times$ Pos & .020 & .023 & 0.87 & 0.76 & .38 \\
\hline & GD & Valence & -.005 & .017 & -0.27 & 0.17 & .68 \\
\hline & & Position & .112 & .017 & 6.59 & 89.25 & $<.001$ \\
\hline & & Val $\times$ Pos & .019 & .024 & 0.77 & 0.60 & .44 \\
\hline & TD & Valence & .004 & .019 & 0.22 & 0.001 & .97 \\
\hline & & Position & .084 & .024 & 3.51 & 13.69 & $<.001$ \\
\hline & & Val $\times$ Pos & -.011 & .029 & -0.37 & 0.14 & .71 \\
\hline & & & $b$ & $S E$ & $t$ & $\chi^{2}$ & $p$ \\
\hline \multirow[t]{2}{*}{ Target } & FFD & Valence & .024 & .014 & 1.78 & 2.61 & .11 \\
\hline & & Position & .011 & .012 & 0.88 & 0.07 & .79 \\
\hline
\end{tabular}




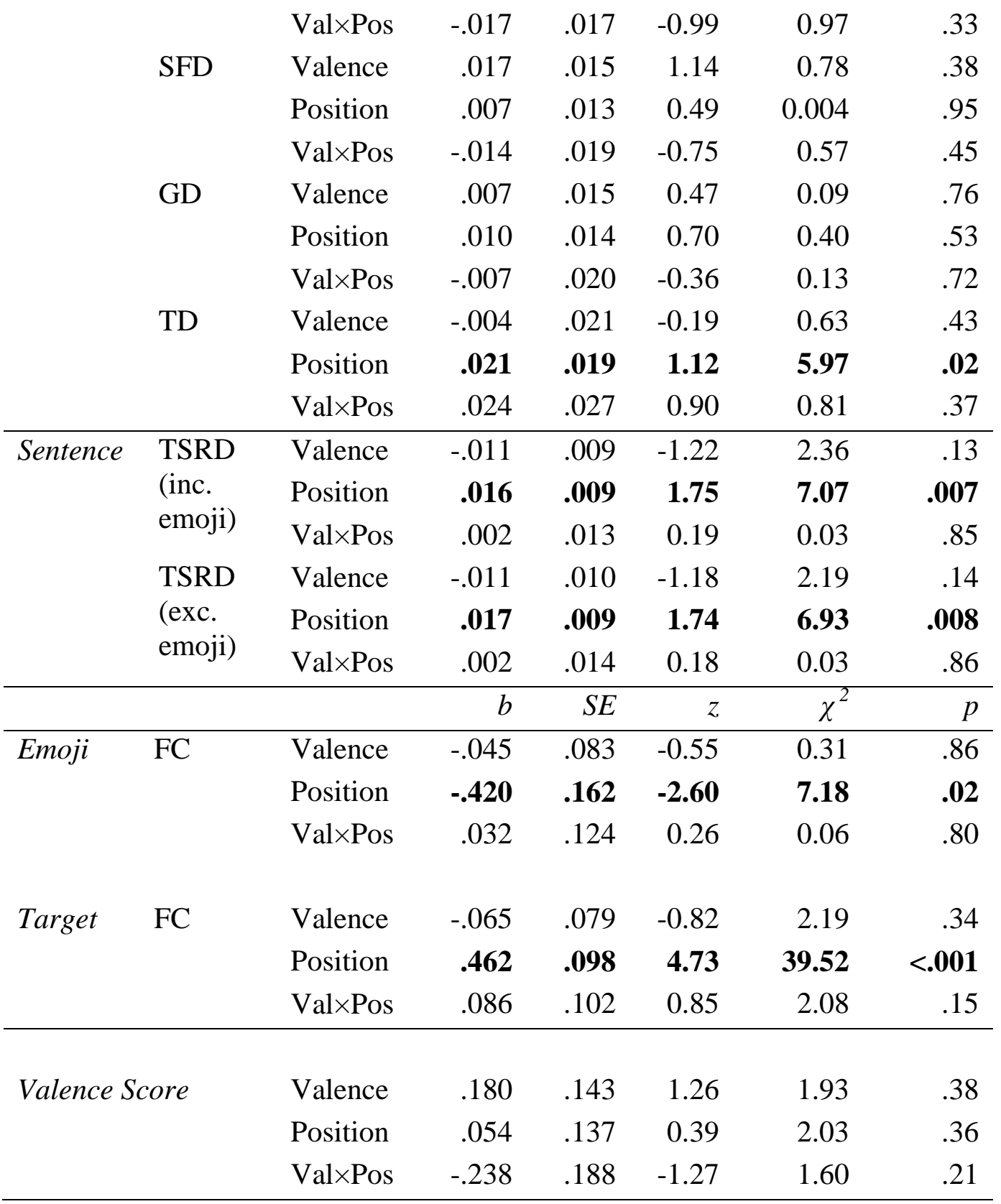

400

401

402

403

404

405

406

407

408

409

Note. $\quad$ LME $=$ linear mixed effects models; Likelihood-Ratio $=$ likelihood-ratio tests; AOI $=$ area of interest; FFD = first fixation duration, SFD = single fixation duration, GD = gaze duration, $\mathrm{TD}=$ total fixation duration, $\mathrm{TSRD}=$ total sentence reading duration, FC = fixation count. Significant fixed effects are highlighted in bold. Statistical models have been generated using logarithm-transformed dependent variables at base 10. $b$-, $S E$ - and $p$-values rounded to 3DP, $z$ and $\chi^{2}$ scores rounded to 2DP.

\subsection{Target Word Region}




\section{EMOJI EFFECTS ON READING AND SENTENCE RATING}

As with the emoji region analyses, the residual plots for the fixation measures within the target word region indicated breaches in residual normality, and as such logarithm transformations were applied at base 10 .

Descriptive statistics are presented in Table 2, and a summary of models and likelihood-ratio tests are presented in Table 3. The results for target region measures did not indicate significant main effects of emoji valence or emoji position on target first-pass reading measures (first fixation duration, single fixation duration and gaze duration), and no significant valence-position interactions. However, significant main effects of emoji position were found on total fixation duration and fixation counts in the target region (see Tables 2 and 3). On average, readers spent longer reading and fixated more on centre-positioned target words when emoji were placed in a sentence-final position than sentence-initial.

Across all nested conditions, early-stage fixations on the target word did not vary substantially from one another to indicate any additional semantic integration or comprehension costs on centre-sentence lexical processing as a result of the emoji expression or placement. However, as late-stage measures incorporate visual regressions (right-to-left eye movements), these effects could demonstrate possible re-reading of the sentence and as a result the target word when readers encounter a sentence-final emoji.

\subsection{Total Sentence Reading Duration}

To assess the global impact of emoji valence and position at a sentence processing level, fixations on all words in the sentence were summed to form total sentence reading durations. Two separate sentence reading measures were computed; one that included fixations in the emoji region, and one that excluded them. As with previous analyses, assessment of residual plots indicated breaches of normality, and therefore logarithm transformations were applied at base 10 to total sentence reading durations. Descriptive 


\section{EMOJI EFFECTS ON READING AND SENTENCE RATING}

442

statistics are presented in Table 2, and a summary of models and likelihood-ratio tests are presented in Table 3 . The results showed statistically significant main effects of emoji position on sentence reading time, both including and excluding the emoji region fixations (see Tables 2 and 3, Figure 2). Readers spent longer reading sentences when emoji were placed at sentence-final positions than sentence-initial, mirroring the word-level effects of emoji position on centre position target words.
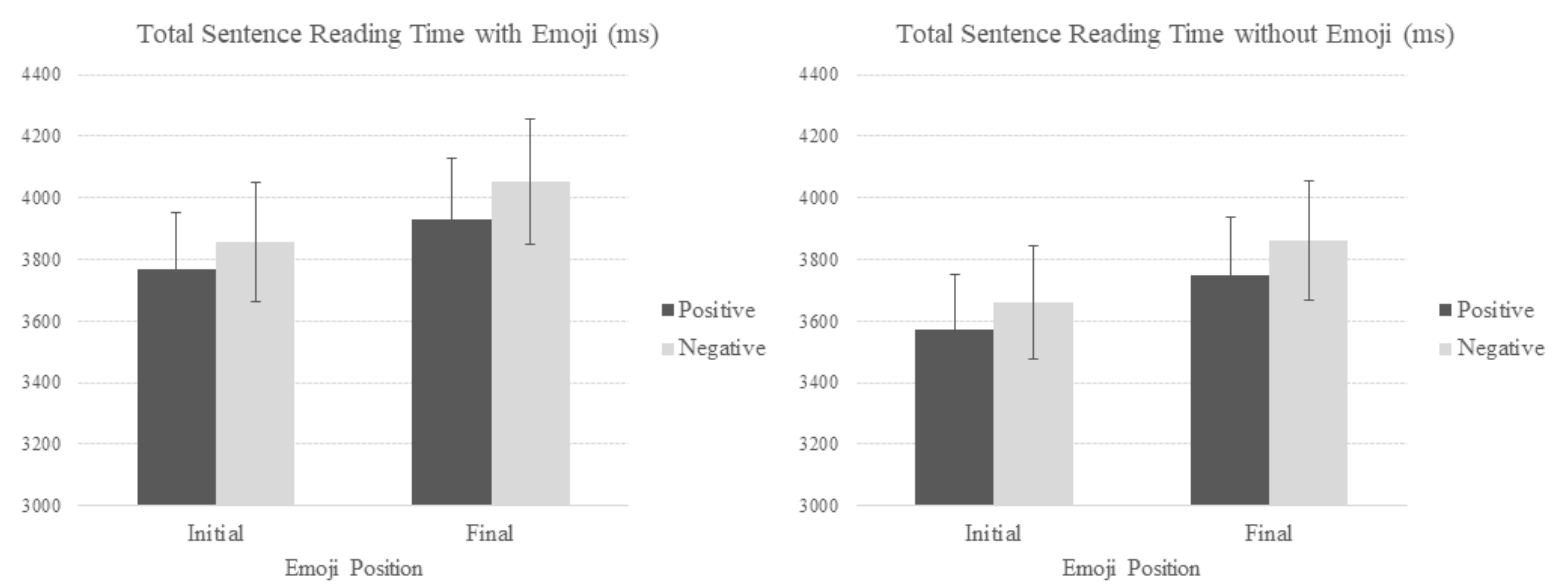

Figure 3. Mean total sentence reading time (with and without emoji region) in positive and negative sentence-initial and sentence-final position conditions.

\subsection{Valence Ratings}

As the perceived emotional valence scores of sentences were classed as ranked data, ordinal response mixed effects models were generated using the 'ordinal' package (Christensen, 2019) using cumulative link mixed modelling. The random effects structure included by-subject random intercepts. Descriptive statistics are presented in Table 2, and a summary of models and likelihood-ratio tests are presented in Table 3. The results showed that the main effects of emoji valence, emoji position and valence-position interaction effects on perceived emotional valence scores were not significant. This suggests participants were 


\section{EMOJI EFFECTS ON READING AND SENTENCE RATING}

not biased by expression on, or position of, the accompanying emoji when interpreting the neutral sentence. In reality, the mean values indicate that scores did not deviate out of the parameters of neutrality, with little difference between conditions.

\section{Discussion}

This study investigated whether the position and valence-related expression of emoji in neutral narrative sentences impacted eye movements during reading and perceptions of emotional valence. Firstly, it was predicted that sentence-final emoji would draw longer fixation durations than emoji in a sentence-initial position. The findings demonstrated consistently longer first-pass and late stage fixations on emoji positioned at the end of the sentence compared to the start, supporting the first prediction. On the other hand, there were also more fixations made on sentence-initial emoji than sentence-final. However, the nonsignificant main effects of emoji valence suggest the emoji expression did not influence fixation durations in the emoji area of interest. Secondly, the research question 'what are the effects of emoji valence and position on centre-position words and sentence reading times?' was addressed. On sentence-level measures, analyses on total sentence reading time showed longer reading times when emoji were in the sentence-final position. On word-level measures, centre position target words had longer total fixation durations and higher fixation counts when emoji were sentence-final. There were no significant effects of emoji position or valence on first-pass fixation measures on target words. Finally, in assessing the research question 'what are the effects of emoji position and valence on perceptions of emotional valence?', no significant main effects on ratings of perceived emotional valence were found. A number of implications can be proposed on the basis of these findings. The emojiposition effects seen in the emoji areas of interest resemble findings from word-position effects (Hirotani et al., 2006; Kuperman et al., 2010; Warren et al., 2009). Previous eye 


\section{EMOJI EFFECTS ON READING AND SENTENCE RATING}

tracking research has demonstrated that words positioned towards the end of the sentence incur an accumulated cost in cognition as a result of 'wrap-up' processes, in which late-stage comprehension and semantic integration occurs once the reader has progressed through the sentence. In this case, it was not a word but an emoji that incurred a cost in the sentence-final position. An explanation for this is that the cost in the processing of the sentence-final emoji is a result of integration of the emoji into the semantic context of the prior sentence, whereas sentence-initial emoji are not impacted by the incremental cost of sentence processing as it is the first thing the reader perceives in the line. However, analysis of the fixation counts in the emoji region showed more fixations were made on sentence-initial emoji than sentence-final. One explanation for this is that sentence-initial placements of emoji are not common, and as such the increased number of fixations on them reflects the novelty of their position. However, if this were the case, this would have also been seen in the fixation duration findings in the form of longer first-pass fixations. On the other hand, it is possible readers regressed back and made short fixations on the sentence-initial emoji to assist in semantic binding processes at later stages of sentence processing. This would imply that sentenceinitial emoji actually make semantic integration more difficult for the reader during sentence wrap-up. It is possible that the predominant positioning of sentence-final emoji in the real world serves a function in cognition, as it allows readers more efficiency in the decoding of emoji meaning during higher-order processing of the sentence. These findings provide fascinating insights into how readers incorporate and integrate digital facial representations, such as emoji, into the lexical processing of an accompanying textual sentence, and have implications for psycholinguistic theory.

In the present study, when assessing target word fixations, emoji position did not affect first-pass processing on the centre-position target word. However, the significant effect of emoji position on late stage fixation measures, which incorporate regressive eye 


\section{EMOJI EFFECTS ON READING AND SENTENCE RATING}

505

506

507

508

509

510

511

512

513

514

515

516

517

518

519

520

521

522

523

524

525

526

527

528

529

movements and refixations on the target words, suggests that readers are more likely to regress to earlier parts of the sentence once they encounter emoji at the end of the sentence.

Whilst the mean differences for target word total fixation durations were arguably small, they are supported by the global sentence-level measures of total sentence reading time, which also demonstrated probable re-reading of the sentence in sentence-final emoji conditions as indicated by the longer summed fixations.

Whilst previous research investigating the effects of facial representations on the perceived emotional valence of the accompanying message has been inconsistent, results have suggested that the inclusion of digital faces has some degree of influence over perceptions of message valence. However, the results from the ordinal mixed-effects models in this study showed no significant differences in emotional valence ratings between conditions, refuting the findings of Derks et al. (2008) and Lo (2008). Given the clear contradictions between the prior and present findings, future research should focus on addressing and investigating why these inconsistent results are occurring. One notable difference between the present study and those stated above is the use of narrative sentences, as opposed to those demonstrating conversational interactions between two or more individuals (Willoughby \& Liu, 2018). Previous findings have demonstrated that contextual factors are important in the applications and usages of emoji (Derks, Bos, \& Von Grumbkow, 2007). It could be that the narrative nature of the sentence resulted in readers disregarding the emoji because they appeared in a somewhat novel context. This would support theoretical suggestions from the Social Information Processing theory (Walther, 1992) that emoji as emotional cues are used for the function of forming and maintaining relationships during communication. As narrative text written in third-person does not have a notable correspondent, readers may disregard the impact of the emoji in favour of the sentence. As such, it is plausible that the linguistic formatting and delivery of the sentence (e.g. narrative 


\section{EMOJI EFFECTS ON READING AND SENTENCE RATING}

point of view; Salem, Weskott, \& Holler, 2017) could moderate the impact of the facial representation on the perception of the accompanying message during experimental trials. Although further replication is needed to confirm this, organisations utilising emoji in a more impersonal context should assess the impact their usage is truly having.

However, another possible explanation is that the sentences used in this study were too neutral for emoji to influence reader perceptions. If longer fixations on sentence-final emoji are linked to wrap-up processes, and thereby the binding of semantic information, it could be that this includes a decision-making process regarding the overall judgements of the message. If the emoji corresponds to the general sentiment of the message, it may boost perceived emotionality; alternatively, an incongruence of the emoji and sentence sentiment could result in perceptions of irony and sarcasm (Thompson \& Filik, 2016). However, if the message is completely neutral, readers may attempt to incorporate the emoji during semantic binding but ultimately decide that the emoji does not add to perceptions of the message. This would have interesting ramifications, as it would indicate that emoji will not automatically boost any type of message they are presented with, and could explain the inconsistencies in research findings, as experimental studies in this area often incorporate specially constructed stimuli. Likewise, if target words in these sentences were strongly semantically emotional (see Scott, O’Donnell, \& Sereno, 2012) and were placed with a positive- or negative-valence context, it could be that first-pass fixations on centre-position target words are affected by both the position and the valence of accompanying facial representations in a similar manner to semantic priming effects (Comesaña et al., 2013). On the other hand, in the present study readers were aware they were going to be required to make valence judgements before seeing the sentences. If readers were presented with the rating task after the delivery of the sentences, which would delay the decision-making process to post-reading, there may be 


\section{EMOJI EFFECTS ON READING AND SENTENCE RATING}

differing effects from the ones found presently. These factors are aspects that should be considered and explored in future research.

The current study was not without certain limitations which should be noted. Firstly, although within the parameters for similar eye-tracking research (Filik, Brightman, Gathercole, \& Leuthold, 2017; Hand, O’Donnell, \& Sereno, 2012; Scott \& Hand, 2016), the sample size in the present study was small and predominantly undergraduate students, which limits the generalizability of these findings. Similarly, the sample was comprised of relatively young individuals, with self-report measures indicating a relatively high usage and exposure to emoji. Although research relating to the age differences in emoji use have been inconsistent (Jaeger et al., 2018; Prada et al., 2018), future replications of this design should consider the potential emoji fluency of readers by utilising a wider range and demographic of participants. Secondly, the use of a chin rest and monitor presentation meant that participant reading behaviours may be regarded as artificial in comparison to contexts in which emoji more often appear (e.g., mobile technology). Although these instruments were a necessity for precise measurement and experimental control, future researchers should explore more natural methods of assessing reading, such as the use of eye trackers allowing for unrestricted head movements and stimulus presentation on a mobile device such as a smart phone.

Similarly, replications should aim to expand on these eye movement findings by exploring alternative on-line measures, such as event-related potentials, to measure emoji effects on sentence wrap-up (see Friedman, Simson, Ritter, \& Rapin, 1975; Hagoort, 2003). Finally, future research should consider expanding on and using a wider variety of emoji than used in this paradigm, which only presented smiling and frowning faces. If the emoji were only slightly valent, it could also contribute to the lack of findings regarding perceived emotional valence in the current study. More expressive faces, such as the grinning $(\ddot{\bullet})$, crying $(\because)$ or angry emoji ( (־)), may elicit greater responses. 


\section{EMOJI EFFECTS ON READING AND SENTENCE RATING}

579

580

581

582

583

584

585

586

587

588

589

590

591

592

593

594

595

596

In conclusion, this study presents the first example of eye movement measures during the reading of narrative sentences containing emoji. The findings demonstrate that emoji positioned at the end of the sentence incur an additional cost when the reader reaches the emoji itself. These costs can be likened to 'wrap-up' costs in the visual processing of words, in which late-stage global semantic integration and comprehension occur (Kuperman et al., 2010; Warren et al., 2009). The effects extended to local- and global-level late stage reading, with longer total reading times on target words and sentences when emoji were at the end of the sentence. However, this was not seen in first-pass reading of centre-positioned target words. On the other hand, an unexpected finding was that emoji did not influence readers' perceptions of the emotionality of the sentence. The authors of this manuscript propose this could be affected by the linguistic characteristics of the sentence (e.g., narrative formatting), which may moderate the impact of the emoji on the perceptions of the accompanying text. This study provides two clear contributions to the literature: firstly, theoretical understanding of higher-order processes such as sentence wrap-up can be extended from words as lexical units to emoji positioned at the end of the sentence. Secondly, the degree of effects that emoji have on the perception formation of accompanying text may be contingent on other linguistic and social factors. These findings provide valuable insights into the integration of emoji in linguistic processing which require further investigation. 


\section{References}

Amaghlobeli, N. (2012). Linguistic Features of Typographic Emoticons in SMS Discourse. Theory and Practice in Language Studies, 2(2), 348-354. https://doi.org/10.4304/tpls.2.2.348-354

Balogh, J., Zurif, E., Prather, P., Swinney, D., \& Finkel, L. (1998). Gap-filling and end-ofsentence effects in real-time language processing: Implications for modeling sentence comprehension in aphasia. Brain and Language, 61(2), 169-182. https://doi.org/10.1006/brln.1997.1917

Bates, D., Mächler, M., Bolker, B., \& Walker, S. (2015). Fitting Linear Mixed-Effects Models Using lme4. Journal of Statistical Software, 67(1). https://doi.org/10.18637/jss.v067.i01

Christensen, R. H. B. (2019). ordinal - Regression Models for Ordinal Data. R package version 2019.4-25. Retrieved from https://cran.rproject.org/web/packages/ordinal/index.html

Comesaña, M., Soares, A. P., Perea, M., Piñeiro, A. P., Fraga, I., \& Pinheiro, A. (2013). ERP correlates of masked affective priming with emoticons. Computers in Human Behavior, 29(3), 588-595. https://doi.org/https://doi.org/10.1016/j.chb.2012.10.020

Derks, D., Bos, A. E. R., \& Von Grumbkow, J. (2007). Emoticons and social interaction on the Internet: the importance of social context. Computers in Human Behavior, 23(1), 842-849. https://doi.org/10.1016/j.chb.2004.11.013

Derks, D., Bos, A. E. R., \& Von Grumbkow, J. (2008). Emoticons and online message interpretation. Social Science Computer Review, 26(3), 379-388. https://doi.org/10.1177/0894439307311611

Filik, R., Brightman, E., Gathercole, C., \& Leuthold, H. (2017). The emotional impact of verbal irony: Eye-tracking evidence for a two-stage process. Journal of Memory and Language, 93, 193-202. https://doi.org/10.1016/j.jml.2016.09.006

Filik, R., Țurcan, A., Thompson, D., Harvey, N., Davies, H., \& Turner, A. (2016). Sarcasm and emoticons: Comprehension and emotional impact. Quarterly Journal of Experimental Psychology, 69(11), 2130-2146. https://doi.org/10.1080/17470218.2015.1106566

Friedman, D., Simson, R., Ritter, W., \& Rapin, I. (1975). The late positive component (P300) and information processing in sentences. Electroencephalography and Clinical Neurophysiology, 38(3), 255-262. https://doi.org/10.1016/0013-4694(75)90246-1

Fullwood, C., Orchard, L. J., \& Floyd, S. A. (2013). Emoticon convergence in Internet chat rooms. Social Semiotics, 23(5), 648-662. https://doi.org/10.1080/10350330.2012.739000

Garrison, A., Remley, D., Thomas, P., \& Wierszewski, E. (2011). Conventional faces: Emoticons in instant messaging discourse. Computers and Composition, 28(2), 112125. https://doi.org/https://doi.org/10.1016/j.compcom.2011.04.001

Gawne, L., \& McCulloch, G. (2019). Emoji as digital gestures. Language@Internet, 17(2). Retrieved from https://www.languageatinternet.org/articles/2019/gawne

Hagoort, P. (2003). How the brain solves the binding problem for language: a neurocomputational model of syntactic processing. NeuroImage, 20(5-6), 18-29. https://doi.org/10.1016/j.neuroimage.2003.09.013 
Hand, C. J., Miellet, S., O’Donnell, P. J., \& Sereno, S. C. (2010). The frequencypredictability interaction in reading: It depends where you're coming from. Journal of Experimental Psychology: Human Perception and Performance, 36(5), 1294-1313. https://doi.org/10.1037/a0020363

Hand, C. J., O’Donnell, P. J., \& Sereno, S. C. (2012). Word-Initial Letters Influence Fixation Durations during Fluent Reading. Frontiers in Psychology, 3(APR), 1-19. https://doi.org/10.3389/fpsyg.2012.00085

Hirotani, M., Frazier, L., \& Rayner, K. (2006). Punctuation and intonation effects on clause and sentence wrap-up: Evidence from eye movements. Journal of Memory and Language, 54(3), 425-443. https://doi.org/10.1016/j.jml.2005.12.001

Jaeger, S. R., Xia, Y., Lee, P. Y., Hunter, D. C., Beresford, M. K., \& Ares, G. (2018). Emoji questionnaires can be used with a range of population segments: Findings relating to age, gender and frequency of emoji/emoticon use. Food Quality and Preference. https://doi.org/10.1016/j.foodqual.2017.12.011

Kaye, L. K., Malone, S. A., \& Wall, H. J. (2017). Emojis: Insights, Affordances, and Possibilities for Psychological Science. Trends in Cognitive Sciences, 21(2), 66-68. https://doi.org/https://doi.org/10.1016/j.tics.2016.10.007

Kaye, L. K., Wall, H. J., \& Malone, S. A. (2016). "Turn that frown upside-down”: A contextual account of emoticon usage on different virtual platforms. Computers in Human Behavior, 60, 463-467. https://doi.org/10.1016/j.chb.2016.02.088

Kemp, N. (2010). Texting versus txtng: reading and writing text messages, and links with other linguistic skills. Writing Systems Research, 2(1), 53-71.

https://doi.org/10.1093/wsr/wsq002

Kuperman, V., Dambacher, M., Nuthmann, A., \& Kliegl, R. (2010). The effect of word position on eye-movements in sentence and paragraph reading. Quarterly Journal of Experimental Psychology, 63(9), 1838-1857. https://doi.org/10.1080/17470211003602412

Lo, S. (2008). The nonverbal communication functions of emoticons in computer-mediated communication. CyberPsychology \& Behavior, 11(5), 595-597. https://doi.org/10.1089/cpb.2007.0132

Oleszkiewicz, A., Karwowski, M., Pisanski, K., Sorokowski, P., Sobrado, B., \& Sorokowska, A. (2017). Who uses emoticons? Data from 86702 Facebook users. Personality and Individual Differences, 119, 289-295. https://doi.org/10.1016/j.paid.2017.07.034

Payne, B. R., \& Stine-Morrow, E. A. L. (2012). Aging, parafoveal preview, and semantic integration in sentence processing: Testing the cognitive workload of wrap-up. Psychology and Aging, 27(3), 638-649. https://doi.org/10.1037/a0026540

Prada, M., Rodrigues, D. L., Garrido, M. V., Lopes, D., Cavalheiro, B., \& Gaspar, R. (2018). Motives, frequency and attitudes toward emoji and emoticon use. Telematics and Informatics, 35(7), 1925-1934. https://doi.org/10.1016/j.tele.2018.06.005

Rayner, K. (1998). Eye movements in reading and information processing: 20 years of research. Psychological Bulletin, 124(3), 372-422. https://doi.org/10.1037/00332909.124.3.372

Rayner, K. (2009). Eye movements and attention in reading, scene perception, and visual search. Quarterly Journal of Experimental Psychology, 62(8), 1457-1506. 
https://doi.org/10.1080/17470210902816461

Rayner, K., Carlson, M., \& Frazier, L. (1983). The interaction of syntax and semantics during sentence processing: eye movements in the analysis of semantically biased sentences. Journal of Verbal Learning and Verbal Behavior, 22(3), 358-374. https://doi.org/10.1016/S0022-5371(83)90236-0

Reichle, E. D., \& Sheridan, H. (2015). E-Z Reader: An Overview of the Model and Two Recent Applications. In A. Pollatsek \& R. Treiman (Eds.), The Oxford Handbook of Reading (pp. 227-292). Oxford: Oxford University Press. https://doi.org/10.1093/oxfordhb/9780199324576.013.17

Riordan, M. A., \& Kreuz, R. J. (2010). Cues in computer-mediated communication: A corpus analysis. Computers in Human Behavior, 26(6), 1806-1817. https://doi.org/https://doi.org/10.1016/j.chb.2010.07.008

Rodrigues, D., Lopes, D., Prada, M., Thompson, D., \& Garrido, M. V. (2017). A frown emoji can be worth a thousand words: Perceptions of emoji use in text messages exchanged between romantic partners. Telematics and Informatics, 34(8), 1532-1543. https://doi.org/10.1016/j.tele.2017.07.001

Rodríguez-Hidalgo, C., Tan, E. S. H., \& Verlegh, P. W. J. (2017). Expressing emotions in blogs: The role of textual paralinguistic cues in online venting and social sharing posts. Computers in Human Behavior, 73, 638-649. https://doi.org/https://doi.org/10.1016/j.chb.2017.04.007

Salem, S., Weskott, T., \& Holler, A. (2017). Does narrative perspective influence readers' perspective-taking? An empirical study on free indirect discourse, psycho-narration and first-person narration. Glossa: A Journal of General Linguistics, 2(1), 61. https://doi.org/10.5334/gjgl.225

Scott, G. G., \& Hand, C. J. (2016). Motivation determines Facebook viewing strategy: An eye movement analysis. Computers in Human Behavior. https://doi.org/10.1016/j.chb.2015.11.029

Scott, G. G., Keitel, A., Becirspahic, M., Yao, B., \& Sereno, S. C. (2019). The Glasgow Norms: Ratings of 5,500 words on nine scales. Behavior Research Methods, 51(3), 1258-1270. https://doi.org/10.3758/s13428-018-1099-3

Scott, G. G., O’Donnell, P. J., \& Sereno, S. C. (2012). Emotion words affect eye fixations during reading. Journal of Experimental Psychology: Learning Memory and Cognition, 38(3), 783-792. https://doi.org/10.1037/a0027209

Sereno, S. C., \& Rayner, K. (2003). Measuring word recognition in reading: Eye movements and event-related potentials. Trends in Cognitive Sciences, 7(11), 489-493.

https://doi.org/10.1016/j.tics.2003.09.010

Skovholt, K., Grønning, A., \& Kankaanranta, A. (2014). The communicative functions of emoticons in workplace e-mails: :-). Journal of Computer-Mediated Communication, 19(4), 780-797. https://doi.org/10.1111/jcc4.12063

Spina, S. (2018). Role of Emoticons as Structural Markers in Twitter Interactions. Discourse Processes, 56(4), 345-362. https://doi.org/10.1080/0163853X.2018.1510654

Tauch, C., \& Kanjo, E. (2016). The roles of emojis in mobile phone notifications. In Proceedings of the 2016 ACM International Joint Conference on Pervasive and Ubiquitous Computing Adjunct - UbiComp '16 (pp. 1560-1565). 
https://doi.org/10.1145/2968219.2968549

Thompson, D., \& Filik, R. (2016). Sarcasm in written communication: Emoticons are efficient markers of intention. Journal of Computer-Mediated Communication, 21(2), 105-120. https://doi.org/10.1111/jcc4.12156

Thompson, D., Mackenzie, I. G., Leuthold, H., \& Filik, R. (2016). Emotional responses to irony and emoticons in written language: Evidence from EDA and facial EMG. Psychophysiology, 53(7), 1054-1062. https://doi.org/10.1111/psyp.12642

van Heuven, W. J. B., Mandera, P., Keuleers, E., \& Brysbaert, M. (2014). SUBTLEX-UK: A new and improved word frequency database for British English. Quarterly Journal of Experimental Psychology, 67(6), 1176-1190. https://doi.org/10.1080/17470218.2013.850521

Walther, J. B. (1992). Interpersonal Effects in Computer-Mediated Interaction. Communication Research, 19(1), 52-90. https://doi.org/10.1177/009365092019001003

Walther, J. B., \& D'Addario, K. P. (2001). The impacts of emoticons on message interpretation in computer-mediated communication. Social Science Computer Review, 19(3), 324-347. https://doi.org/10.1177/089443930101900307

Warren, T., White, S. J., \& Reichle, E. D. (2009). Investigating the causes of wrap-up effects: Evidence from eye movements and E-Z Reader. Cognition, 111(1), 132-137. https://doi.org/10.1016/j.cognition.2008.12.011

Weissman, B., \& Tanner, D. (2018). A strong wink between verbal and emoji-based irony: How the brain processes ironic emojis during language comprehension. PLOS ONE, 13(8), 1-26. https://doi.org/10.1371/journal.pone.0201727

Willoughby, J. F., \& Liu, S. (2018). Do pictures help tell the story? An experimental test of narrative and emojis in a health text message intervention. Computers in Human Behavior, 79, 75-82. https://doi.org/10.1016/j.chb.2017.10.031

Wolf, A. (2000). Emotional expression online: Gender differences in emoticon use. CyberPsychology \& Behavior, 3(5), 827-833. https://doi.org/10.1089/10949310050191809 


\section{Appendix A}

Experimental sentence stimuli and target word characteristics

\begin{tabular}{|c|c|c|c|c|c|c|}
\hline $\begin{array}{l}\text { Target } \\
\text { Word }\end{array}$ & $\underline{\text { Sentence }}$ & $\frac{\text { Sentence }}{\underline{\text { Length }}}$ & $\underline{\text { Target }}$ & $\frac{\text { Target }}{\text { Valence }}$ & $\frac{\text { SUBTLEX- }}{\underline{\text { UK Freq }}}$ & 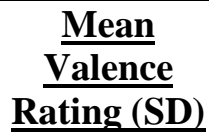 \\
\hline beach & Yasmin thought the weather at the beach was warm for this time of year & 70 & 3.49 & 5.24 & 10594 & $5.94(1.19)$ \\
\hline brief & The ten year school reunion was a brief meeting with some old friends & 69 & 3.24 & 5.03 & 3647 & $5.03(1.23)$ \\
\hline chest & When Harry looked down at his chest he saw something sitting on his shirt & 73 & 5.68 & 5.29 & 6105 & $4.19(1.14)$ \\
\hline chief & At the press conference the chief told the public the result of the enquiry & 75 & 4.83 & 5.97 & 12548 & $4.75(0.97)$ \\
\hline child & The mother looked down at her child to see if he was doing his homework & 71 & 4.23 & 6.19 & 28117 & $5.19(0.92)$ \\
\hline coast & Sandy stood on the pier next to the coast and waited for her boyfriend & 70 & 5.58 & 7.28 & 14755 & $5.67(1.09)$ \\
\hline court & When the lawyer left the crown court they sat and considered the outcome & 72 & 3.97 & 4.09 & 25553 & $4.67(0.96)$ \\
\hline crowd & The man walked through the crowd at the concert looking for his partner & 71 & 5.21 & 4.76 & 11702 & $4.83(1.36)$ \\
\hline dream & Joshua sat in his bed after a dream woke him up during the night time & 69 & 6.30 & 7.41 & 19951 & $4.19(1.19)$ \\
\hline field & While the farmer harvested the field his wife fed the farm animals & 66 & 3.70 & 5.38 & 16714 & $5.69(1.04)$ \\
\hline horse & The police officer sat on her horse and noticed the group walking by & 68 & 4.87 & 6.07 & 19690 & $5.06(0.63)$ \\
\hline hotel & When the guest returned to the hotel later there was nobody to be seen & 70 & 5.28 & 5.91 & 11614 & $4.33(1.01)$ \\
\hline judge & The jury returned and told the judge their decision on the verdict & 66 & 4.97 & 4.82 & 9781 & $4.53(0.94)$ \\
\hline light & The librarian turned on the light in the reading room when it went dark & 71 & 5.66 & 7.19 & 38200 & $5.56(1.11)$ \\
\hline metal & When Jane looked closely at the metal box she could see some markings & 69 & 4.50 & 5.10 & 8629 & $5.14(0.83)$ \\
\hline music & Even though the DJ had downloaded music he bought records almost daily & 70 & 7.77 & 7.97 & 44883 & $5.81(0.95)$ \\
\hline order & Steven waited at home for his order to be delivered in the morning & 66 & N/A & N/A & 28500 & $5.19(1.14)$ \\
\hline
\end{tabular}




\section{EMOJI EFFECTS ON READING AND SENTENCE RATING}

\begin{tabular}{|c|c|c|c|c|c|c|}
\hline paper & The worker left to buy more paper from the stationary store for the office & 74 & 3.66 & 5.65 & 17429 & $4.92(0.77)$ \\
\hline party & Jenny started dressing for the party when there was a knock on the door & 71 & 7.56 & 8.15 & 67729 & $4.75(0.91)$ \\
\hline phone & Jerry had checked his new phone earlier on the train station platform & 69 & 4.56 & 5.36 & 31257 & $5.36(0.96)$ \\
\hline piano & Peter was practicing on his piano before his recital the following day & 70 & 5.60 & 6.42 & 4610 & $5.83(1.46)$ \\
\hline plant & The biologist watered the green plant sitting on the laboratory shelf & 69 & 4.32 & 6.50 & 12965 & $5.92(1.21)$ \\
\hline radio & Michael turned on his digital radio to listen to the latest broadcast & 69 & 5.06 & 6.06 & 13347 & $5.75(0.84)$ \\
\hline space & The astronaut's training to go to space was pushing them to the limit & 69 & 6.77 & 6.50 & 39102 & $4.67(1.37)$ \\
\hline stage & The director walked onto the stage and reminded the actor of their lines & 72 & 5.23 & 5.52 & 31143 & $4.39(1.34)$ \\
\hline stone & The explorer looked up at the large stone blocking the path to the cave & 71 & 3.34 & 5.06 & 13847 & $4.14(1.29)$ \\
\hline story & Shannon decided to take her story to the publisher to examine once more & 71 & 5.48 & 6.71 & 53104 & $5.06(0.89)$ \\
\hline study & Charlotte returned to her private study and started working on her essay & 72 & 3.74 & 5.43 & 8222 & $5.53(1.28)$ \\
\hline table & The carpenter finished the table and looked at it with a critical eye & 69 & 3.17 & 5.20 & 25504 & $5.69(1.17)$ \\
\hline taste & The gourmet considered the taste of his meal when writing his review & 68 & 6.18 & 6.88 & 17833 & $5.58(0.81)$ \\
\hline theme & When her friends proposed the theme for the party Willow was surprised & 70 & 4.70 & 5.52 & 6385 & $5.28(1.00)$ \\
\hline today & The blogger had other plans for today but instead he played video games & 71 & N/A & $\mathrm{N} / \mathrm{A}$ & 183044 & $4.69(1.28)$ \\
\hline trade & Lisa was hoping to organise a trade of her board game for her friends & 69 & 3.85 & 5.68 & 13771 & $5.17(1.11)$ \\
\hline truck & The shop attendant watched as the truck pulled into the petrol station & 70 & 3.68 & 4.71 & 3314 & $4.92(0.55)$ \\
\hline voice & The sound of the public speaker's voice made the audience fall silent & 69 & 5.63 & 5.97 & 15535 & $5.64(1.29)$ \\
\hline wheel & Jodie went to buy a new bike wheel as the original one had worn out & 67 & 3.60 & 5.43 & 6472 & $5.58(1.20)$ \\
\hline
\end{tabular}

Note. Target arousal and valence taken from The Glasgow Norms (Scott et al., 2019). SUBTLEX-UK frequency of occurrences in UK subtitles (http://crr.ugent.be/archives/1423). Respondents for valence ratings: $N=62$. Standard deviation of mean valence ratings presented in brackets. 
RUNNING HEAD: EMOJI EFFECTS ON READING AND SENTENCE RATING

\section{Appendix B}

Summary of linear mixed effects models random effects structures

We utilised a data driven approach to estimating the random effects structures of our linear mixed effects models. This involved computing a series of random-intercepts and random-slopes models and applying model comparison techniques to assess model convergence and the inclusion of appropriate random effects in the full models. Subjects ( $\mathrm{subj}$ ) and items (item) were treated as random effects, while the fixed factors were the two independent variables, emoji valence and position. The maximal random effects structure included both by-items and by-subjects intercepts, and all possible fixed factor by-items and by-subjects slopes. Table B1 below outlines the random effects structure used in the linear mixed effects models for each respective outcome.

Table B1: Summary of random effects structure

\begin{tabular}{ll}
\hline Measure & Random effects structure \\
\hline Emoji first fixation duration & $(1 \mid \mathrm{subj})$ \\
Emoji single fixation duration & $(1 \mid \mathrm{subj})$ \\
Emoji gaze duration & $(1 \mid$ subj $)$ \\
Emoji total duration & $(1+$ Emoji Position|subj $)$ \\
Emoji fixation count & $(1+$ Emoji Position|subj $)$ \\
Target first fixation duration & $(1 \mid \mathrm{subj})+(1 \mid$ item $)$ \\
Target single fixation duration & $(1 \mid \mathrm{subj})$
\end{tabular}




\title{
EMOJI EFFECTS ON READING AND SENTENCE RATING
}

\author{
Target gaze duration $\quad(1 \mid$ subj $)+(1 \mid$ item $)$ \\ Target total duration $\quad(1 \mid$ subj $)+(1 \mid$ item $)$ \\ Target fixation count $\quad(1+$ Emoji Position|item $)$ \\ Total sentence reading duration (with emoji) $\quad(1 \mid$ subj $)+(1 \mid$ item $)$ \\ Total sentence reading duration (without emoji) $\quad(1 \mid$ subj $)+(1 \mid$ item $)$ \\ Emotional valence rating task $\quad(1+$ Emoji Position + Emoji Valence|subj $)+(1+$ Emoji Position + Emoji Valence $\mid$ item $)$
}

Note. Value 1 represents inclusion of random intercept. 
Christopher Robus: Conceptualisation, Methodology, Software, Formal Analysis, Investigation, Resources, Data Curation, Writing - Original Draft, Writing - Review and Editing, Project Administration.

Christopher Hand: Formal Analysis, Resources, Data Curation, Writing - Original Draft, Writing Review and Editing, visualisation

Ruth Filik: Methodology, Writing - Original Draft, Writing - Review and Editing Melanie Pitchford: Supervision, Writing - Original Draft, Writing - Review and Editing 